\title{
Asymptotics of eigenvalues for Sturm-Liouville problem including eigenparameter-dependent boundary conditions with integrable potential
}

\author{
Elif Baskaya \\ Department of Mathematics, Karadeniz Technical University, Trabzon, Turkey
}

Received: 16 December 2017, Accepted: 22 January 2018

Published online: 22 July 2018.

\begin{abstract}
In this paper, we obtain asymptotic estimates of eigenvalues for regular Sturm-Liouville problems having the eigenvalue parameter in all boundary conditions with integrable potential.
\end{abstract}

Keywords: Sturm-Liouville problems, integrable potential, eigenvalues, asymptotics.

\section{Introduction}

In this paper, we consider the boundary value problem

$$
\begin{aligned}
& y^{\prime \prime}(t)+\{\lambda-q(t)\} y(t)=0, t \in[a, b], \\
& a_{1} y(a)+a_{2} y^{\prime}(a)=\lambda\left[a_{1}^{\prime} y(a)+a_{2}^{\prime} y^{\prime}(a)\right], \\
& b_{1} y(b)+b_{2} y^{\prime}(b)=\lambda\left[b_{1}^{\prime} y(b)+b_{2}^{\prime} y^{\prime}(b)\right],
\end{aligned}
$$

where $\lambda$ is a real parameter; $q(t)$ is a real-valued function; $a_{i}, a_{i}^{\prime}, b_{i}, b_{i}^{\prime} \in \mathbb{R}, i=0,1$. Also we assume that $q(t)$ is integrable. This problem differs from the usual regular Sturm-Liouville problem in the sense that eigenvalue parameter $\lambda$ is contained in the boundary condition at $a$. Problems of this type arise from the method of separation of variables applied to mathematical models for certain physical problems including heat conduction and wave propagation, etc. [8]. It is shown by Walter [15] that this problem is self-adjoint problem. The purpose of this paper is to obtain asymptotic approximations for the eigenvalues of (1)-(3).

Approximations of this type have been derived before. We mention in particular [7,8] and [2]. Fulton's approach in [7] is based on an iteration of the usual Volterra integral equation, producing an asymptotic expansion of the solution in higher powers of $1 / \lambda^{1 / 2}$ as $\lambda \rightarrow \infty$ and in [8] is based on the analysis of [14] for regular Sturm-Liouville problems on a finite closed interval and involves some operator-theoretical results of [15]. The approach used in [2] is based on an iterative procedure solving the associated Riccati equation and producing an asymptotic expansion of the solution in the higher powers of $1 / \lambda^{1 / 2}$ as $\lambda \rightarrow \infty$ for smooth $q(t)$. There is also a vast amount of literature dealing with asymptotic estimates of eigenvalues for standart Sturm-Liouville problems with regular endpoints [3,4,5,6,9,10,11,13,14]. Here we follow the similar approach in $[4,10,12]$. We assume without loss of generality, that $q(t)$ has mean value zero. That is $\int_{a}^{b} q(t) d t=0$ 


\section{Conclusion}

Theorem 1. The eigenvalues $\lambda_{n}$ of (1)-(3) satisfy as $\lambda \rightarrow \infty$,

(i) if $a_{2}^{\prime} \neq 0$ and $b_{2}^{\prime} \neq 0$,

$$
\begin{aligned}
\lambda_{n}^{1 / 2}= & \frac{(n+1) \pi}{(b-a)}+\frac{1}{(n+1) \pi}\left\{\frac{a_{2}^{\prime} b_{1}^{\prime}-a_{1}^{\prime} b_{2}^{\prime}}{a_{2}^{\prime} b_{2}^{\prime}}+\frac{1}{2} \int_{a}^{b}\left(\cos \frac{2(n+1) \pi(x-a)}{b-a}\right) q(x) d x\right\} \\
& +O\left(n^{-2} \eta(n)\right)+O\left(n^{-1} \eta^{2}(n)\right),
\end{aligned}
$$

(ii) if $a_{2}^{\prime} \neq 0$ and $b_{2}^{\prime}=0$,

$$
\begin{aligned}
\lambda_{n}^{1 / 2}= & \frac{(2 n+3) \pi}{2(b-a)}+\frac{2}{(2 n+3) \pi}\left\{\frac{a_{2}^{\prime} b_{2}-a_{1}^{\prime} b_{1}^{\prime}}{a_{2}^{\prime} b_{1}^{\prime}}+\frac{1}{2} \int_{a}^{b}\left(\cos \frac{(2 n+3) \pi(x-a)}{b-a}\right) q(x) d x\right\} \\
& +O\left(n^{-2} \eta(n)\right)+O\left(n^{-1} \eta^{2}(n)\right) .
\end{aligned}
$$

Theorem 2. The eigenvalues $\lambda_{n}$ of (1)-(3) satisfy as $\lambda \rightarrow \infty$,

(i) if $a_{2}^{\prime}=0$ and $b_{2}^{\prime} \neq 0$,

$$
\begin{aligned}
\lambda_{n}^{1 / 2}= & \frac{(2 n+3) \pi}{2(b-a)}+\frac{2}{(2 n+3) \pi}\left\{\frac{a_{1}^{\prime} b_{1}^{\prime}-a_{2} b_{2}^{\prime}}{a_{1}^{\prime} b_{2}^{\prime}}-\frac{1}{2} \int_{a}^{b}\left(\cos \frac{(2 n+3) \pi(x-a)}{b-a}\right) q(x) d x\right\} \\
& +O\left(n^{-2} \eta(n)\right)+O\left(n^{-1} \eta^{2}(n)\right),
\end{aligned}
$$

(ii) if $a_{2}^{\prime}=0$ and $b_{2}^{\prime}=0$,

$$
\begin{aligned}
\lambda_{n}^{1 / 2}= & \frac{(n+2) \pi}{(b-a)}+\frac{1}{(n+2) \pi}\left\{\frac{a_{1}^{\prime} b_{2}-a_{2} b_{1}^{\prime}}{a_{1}^{\prime} b_{1}^{\prime}}-\frac{1}{2} \int_{a}^{b}\left(\cos \frac{2(n+2) \pi(x-a)}{b-a}\right) q(x) d x\right\} \\
& +O\left(n^{-2} \eta(n)\right)+O\left(n^{-1} \eta^{2}(n)\right) .
\end{aligned}
$$

\section{The method}

We associate with (1) the Riccati equation

$$
v^{\prime}(t, \lambda)=-\lambda+q-v^{2}
$$

We define

$$
\begin{aligned}
S(t, \lambda) & =\operatorname{Re}[v(t, \lambda)], \\
T(t, \lambda) & =\operatorname{Im}[v(t, \lambda)] .
\end{aligned}
$$

It is shown in [3] that any real-valued solution of (1) is in the form

$$
y(t, \lambda)=R(t, \lambda) \cos \theta(t, \lambda)
$$

with

$$
\begin{aligned}
& S(t, \lambda)=\frac{R^{\prime}(t, \lambda)}{R(t, \lambda)}, \\
& T(t, \lambda)=\theta^{\prime}(t, \lambda) .
\end{aligned}
$$


Our approach to calculating $\lambda_{n}$ is to approximate those $\lambda$ which are such that

$$
\theta(b, \lambda)-\theta(a, \lambda)=\int_{a}^{b} T(x, \lambda) d x
$$

We suppose that there exist functions $A(t)$ and $\eta(\lambda)$ so that

$$
\left|\int_{t}^{b} e^{2 i \lambda^{1 / 2} x} q(x) d x\right| \leq A(t) \eta(\lambda), \quad t \in[a, b]
$$

where

(i) $A(t):=\int_{t}^{b}|q(x)| d x$ is a decreasing function of $t$,

(ii) $A(.) \in L[a, b]$,

(iii) $\eta(\lambda) \rightarrow 0$ as $\lambda^{1 / 2} \rightarrow \infty$.

For $q \in L[a, b]$ the existence of the $A$ and $\eta$ functions may be established for $\lambda$ positive as follows. We note that, avoiding the trivial case $\int_{t}^{b}|q(x)| d x=0 .\left|\int_{t}^{b} e^{2 i \lambda^{1 / 2} x} q(x) d x\right| \leq \int_{t}^{b}|q(x)| d x<\infty$ so, if we define

$$
F(t, \lambda):= \begin{cases}\left|\int_{t}^{b} e^{2 i \lambda^{1 / 2} x} q(x) d x\right| / \int_{t}^{b}|q(x)| d x, & \text { if } \int_{t}^{b}|q(x)| d x \neq 0 \\ 0, & \text { if } \int_{t}^{b}|q(x)| d x=0\end{cases}
$$

then $0 \leq F(t, \lambda) \leq 1$ and we set $\eta(\lambda):=\sup _{a \leq t \leq b} F(t, \lambda) . \eta(\lambda)$ is well defined by (11) and $\eta(\lambda) \rightarrow 0$ as $\lambda \rightarrow \infty[12]$.

Our method of approximating a solution of $v^{\prime}(t, \lambda)=-\lambda+q-v^{2}$ on $[a, b]$ is similar to [12], so we set

$$
v(t, \lambda):=i \lambda^{1 / 2}+\sum_{n=1}^{\infty} v_{n}(t, \lambda)
$$

When we put this serie into the Riccati equation and solve differential equations, we hold

$$
\begin{gathered}
v_{1}(t, \lambda)=-e^{-2 i \lambda^{1 / 2} t} \int_{t}^{b} e^{2 i \lambda^{1 / 2} x} q(x) d x, \\
v_{2}(t, \lambda)=e^{-2 i \lambda^{1 / 2} t} \int_{t}^{b} e^{2 i \lambda^{1 / 2} x} v_{1}^{2}(x, \lambda) d x, \\
v_{n}(t, \lambda)=e^{-2 i \lambda^{1 / 2} t} \int_{t}^{b} e^{2 i \lambda^{1 / 2} x}\left[v_{n-1}^{2}(x, \lambda)+2 v_{n-1}(x, \lambda) \sum_{m=1}^{n-2} v_{m}(x, \lambda)\right] d x, \quad n \geq 3 .
\end{gathered}
$$

Also we found $\theta(b, \lambda)-\theta(a, \lambda)=\int_{a}^{b} T(x, \lambda) d x$, so with (8) and (12) we have

$$
\theta(b, \lambda)-\theta(a, \lambda)=\int_{a}^{b}\left[\lambda^{1 / 2}+\operatorname{Im} \sum_{n=1}^{\infty} v_{n}(x, \lambda)\right] d x
$$

then

$$
\theta(b, \lambda)-\theta(a, \lambda)=\lambda^{1 / 2}(b-a)+\sum_{n=1}^{\infty} \operatorname{Im} \int_{a}^{b} v_{n}(x, \lambda) d x .
$$

Theorem 3. [1] If $v(t, \lambda)$ as in (12), as $\lambda \rightarrow \infty$

$$
v(t, \lambda)=i \lambda^{1 / 2}+v_{1}(t, \lambda)+O\left(\eta^{2}(\lambda)\right)
$$


where

$$
v_{1}(t, \lambda)=-e^{-2 i \lambda^{1 / 2} t} \int_{t}^{b} e^{2 i \lambda^{1 / 2} x} q(x) d x=-\left[\cos 2 \lambda^{1 / 2} t-i \sin 2 \lambda^{1 / 2} t\right] \times \int_{t}^{b}\left[\cos 2 \lambda^{1 / 2} x+i \sin 2 \lambda^{1 / 2} x\right] q(x) d x
$$

and $\eta(\lambda)$ is defined (11).

After some calculations by using the last theorem, with (4) we gain

$$
S(t, \lambda)=-\left(\cos 2 \lambda^{1 / 2} t\right) \int_{t}^{b}\left[\cos 2 \lambda^{1 / 2} x\right] q(x) d x-\left(\sin 2 \lambda^{1 / 2} t\right) \int_{t}^{b}\left[\sin 2 \lambda^{1 / 2} x\right] q(x) d x+O\left(\eta^{2}(\lambda)\right) .
$$

Let define the following notations:

$$
\begin{aligned}
\sin \xi_{t} & :=\int_{t}^{b}\left(\cos 2 \lambda^{1 / 2} x\right) q(x) d x, \\
\cos \xi_{t} & :=\int_{t}^{b}\left(\sin 2 \lambda^{1 / 2} x\right) q(x) d x
\end{aligned}
$$

thus we can write $S(t, \lambda)$ as

$$
S(t, \lambda)=-\sin \left(2 \lambda^{1 / 2} t+\xi_{t}\right)+O\left(\eta^{2}(\lambda)\right) .
$$

Similarly, with (5) we find $T(t, \lambda)$ as

$$
T(t, \lambda)=\lambda^{1 / 2}-\cos \left(2 \lambda^{1 / 2} t+\xi_{t}\right)+O\left(\eta^{2}(\lambda)\right) .
$$

Also, by using integration by part to (13), we determine

$$
\int_{a}^{b} v_{1}(x, \lambda) d x=\frac{i}{2 \lambda^{1 / 2}} \int_{a}^{b} e^{2 i \lambda^{1 / 2}(x-a)} q(x) d x
$$

and again with integration by part

$$
\begin{aligned}
\int_{a}^{b} v_{1}(x, \lambda) d x & =\frac{i}{2} \lambda^{-1 / 2}\left[\int_{a}^{b} i q(x)\left[\sin 2 \lambda^{1 / 2} x\right]\left[\cos 2 \lambda^{1 / 2} a\right] d x-\int_{a}^{b} i q(x)\left[\cos 2 \lambda^{1 / 2} x\right]\left[\sin 2 \lambda^{1 / 2} a\right] d x\right. \\
& +\frac{i}{2} \lambda^{-1 / 2}\left[\int_{a}^{b} q(x)\left[\cos 2 \lambda^{1 / 2} x\right]\left[\cos 2 \lambda^{1 / 2} a\right] d x+\int_{a}^{b} q(x)\left[\sin 2 \lambda^{1 / 2} x\right]\left[\sin 2 \lambda^{1 / 2} a\right] d x\right.
\end{aligned}
$$

so

$$
\operatorname{Im} \int_{a}^{b} v_{1}(x, \lambda) d x=\frac{1}{2} \lambda^{-1 / 2}\left[\cos 2 \lambda^{1 / 2} a\right] \int_{a}^{b} q(x)\left[\cos 2 \lambda^{1 / 2} x\right] d x+\left[\sin 2 \lambda^{1 / 2} a\right] \int_{a}^{b} q(x)\left[\sin 2 \lambda^{1 / 2} x\right] d x .
$$

We also have from equation (13),

$$
\int_{a}^{b} v_{2}(x, \lambda) d x=\frac{i}{2 \lambda^{1 / 2}} \int_{a}^{b}\left[1-e^{2 i \lambda^{1 / 2}(x-a)}\right] v_{1}^{2}(x, \lambda) d x
$$

and for $n \geq 3$

$$
\int_{a}^{b} v_{n}(x, \lambda) d x=\frac{i}{2 \lambda^{1 / 2}} \times \int_{a}^{b}\left[1-e^{2 i \lambda^{1 / 2}(x-a)}\right]\left[v_{n-1}^{2}(x, \lambda)+2 v_{n-1}(x, \lambda) \sum_{m=1}^{n-2} v_{m}(x, \lambda)\right] d x .
$$


Thus, with the last equations

$$
\int_{a}^{b} \sum_{n=1}^{\infty} \operatorname{Im}\left\{v_{n}(x, \lambda)\right\} d x=\sum_{n=1}^{\infty} \operatorname{Im}\left\{\int_{a}^{b} v_{n}(x, \lambda) d x\right\}=\frac{1}{2} \lambda^{-1 / 2} \sin \left(2 \lambda^{1 / 2} t+\xi_{t}\right)+O\left(\lambda^{-1} \eta(\lambda)\right) .
$$

\section{Proof of the main results}

\section{Proof of theorem 1.}

(i) If $a_{2}^{\prime} \neq 0$ and $b_{2}^{\prime} \neq 0$, the real solution of $y^{\prime \prime}(t)+[\lambda-q(t)] y(t)=0$ is $y(t, \lambda)=R(t, \lambda) \cos \theta(t, \lambda)$ from (6). We use this equation for boundary $t=a$, we find

$$
R(a, \lambda)\left\{\cos \theta(a, \lambda)\left[\left(-a_{2}^{\prime} \lambda+a_{2}\right) \frac{R^{\prime}(a, \lambda)}{R(a, \lambda)}+\left(-a_{1}^{\prime} \lambda+a_{1}\right)\right]+\left(a_{2}^{\prime} \lambda-a_{2}\right) \theta^{\prime}(a, \lambda) \sin \theta(a, \lambda)\right\}=0 .
$$

If we choose $\alpha_{1}$ as

$$
\begin{aligned}
& \sin \alpha_{1}:=\left(-a_{2}^{\prime} \lambda+a_{2}\right) \frac{R^{\prime}(a, \lambda)}{R(a, \lambda)}+\left(-a_{1}^{\prime} \lambda+a_{1}\right), \\
& \cos \alpha_{1}:=\left(a_{2}^{\prime} \lambda-a_{2}\right) \theta^{\prime}(a, \lambda),
\end{aligned}
$$

we have $R(a, \lambda) \sin \left[\alpha_{1}+\theta(a, \lambda)\right]=0$ so $\sin \left(\alpha_{1}+\theta(a, \lambda)\right)=0$, or $\theta(a, \lambda)=-\alpha_{1}$. Using by equations (7) and (8) as $S(t, \lambda)=\frac{R^{\prime}(t, \lambda)}{R(t, \lambda)}, T(t, \lambda)=\theta^{\prime}(t, \lambda)$ and their asymptotic expansions (15)-(16), we calculate

$$
\begin{gathered}
-a_{1}^{\prime} \lambda+\lambda a_{2}^{\prime} \sin \left(2 \lambda^{1 / 2} a+\xi_{a}\right)+a_{1}-a_{2} \sin \left(2 \lambda^{1 / 2} a+\xi_{a}\right) \\
\frac{\sin \alpha_{1}}{\cos \alpha_{1}}=\frac{O\left(\eta^{2}(\lambda)\right)+O\left(\lambda \eta^{2}(\lambda)\right)}{a_{2}^{\prime} \lambda^{3 / 2}-\lambda a_{2}^{\prime} \cos \left(2 \lambda^{1 / 2} a+\xi_{a}\right)-\lambda^{1 / 2} a_{2}+a_{2} \cos \left(2 \lambda^{1 / 2} a+\xi_{a}\right)} \\
+O\left(\eta^{2}(\lambda)\right)+O\left(\lambda \eta^{2}(\lambda)\right)
\end{gathered}
$$

hence

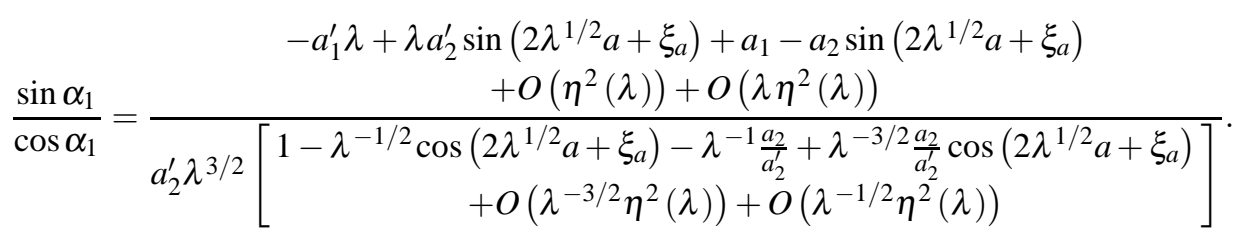

Then

$$
\begin{aligned}
\tan \alpha_{1} & =\left[\begin{array}{c}
-\frac{a_{1}^{\prime}}{a_{2}^{\prime}} \lambda^{-1 / 2}+\lambda^{-1 / 2} \sin \left(2 \lambda^{1 / 2} a+\xi_{a}\right)+\frac{a_{1}}{a_{2}^{\prime}} \lambda^{-3 / 2}-\lambda^{-3 / 2} \frac{a_{2}}{a_{2}^{\prime}} \sin \left(2 \lambda^{1 / 2} a+\xi_{a}\right) \\
+O\left(\lambda^{-1 / 2} \eta^{2}(\lambda)\right)
\end{array}\right] \\
\times & {\left[\begin{array}{c}
1+\lambda^{-1 / 2} \cos \left(2 \lambda^{1 / 2} a+\xi_{a}\right)+\lambda^{-1} \frac{a_{2}}{a_{2}^{\prime}}-\lambda^{-3 / 2} \frac{a_{2}}{a_{2}^{\prime}} \cos \left(2 \lambda^{1 / 2} a+\xi_{a}\right) \\
+O\left(\lambda^{-1 / 2} \eta^{2}(\lambda)\right)
\end{array}\right], }
\end{aligned}
$$

so

$$
\begin{aligned}
\tan \alpha_{1} & =-\frac{a_{1}^{\prime}}{a_{2}^{\prime}} \lambda^{-1 / 2}+\lambda^{-1 / 2} \sin \left(2 \lambda^{1 / 2} a+\xi_{a}\right)-\frac{a_{1}^{\prime}}{a_{2}^{\prime}} \lambda^{-1} \cos \left(2 \lambda^{1 / 2} a+\xi_{a}\right) \\
& +O\left(\lambda^{-1 / 2} \eta^{2}(\lambda)\right) .
\end{aligned}
$$


In the last equation, by using Taylor expansion of $\arctan x$ at $x=0$, we obtain

$$
\alpha_{1}=-\frac{a_{1}^{\prime}}{a_{2}^{\prime}} \lambda^{-1 / 2}+\lambda^{-1 / 2} \sin \left(2 \lambda^{1 / 2} a+\xi_{a}\right)-\frac{a_{1}^{\prime}}{a_{2}^{\prime}} \lambda^{-1} \cos \left(2 \lambda^{1 / 2} a+\xi_{a}\right)+O\left(\lambda^{-1 / 2} \eta^{2}(\lambda)\right)
$$

Similarly, when we use the form $y(t, \lambda)=R(t, \lambda) \cos \theta(t, \lambda)$ for boundary $t=b$, we find

$$
R(b, \lambda)\left\{\cos \theta(b, \lambda)\left[\left(-b_{2}^{\prime} \lambda+b_{2}\right) \frac{R^{\prime}(b, \lambda)}{R(b, \lambda)}+\left(-b_{1}^{\prime} \lambda+b_{1}\right)\right]-\left(-b_{2}^{\prime} \lambda+b_{2}\right) \theta^{\prime}(b, \lambda) \sin \theta(b, \lambda)\right\}=0 .
$$

If we choose $\alpha_{2}$ as

$$
\begin{aligned}
& \sin \alpha_{2}:=\left(-b_{2}^{\prime} \lambda+b_{2}\right) \frac{R^{\prime}(b, \lambda)}{R(b, \lambda)}+\left(-b_{1}^{\prime} \lambda+b_{1}\right), \\
& \cos \alpha_{2}:=\left(-b_{2}^{\prime} \lambda+b_{2}\right) \theta^{\prime}(b, \lambda),
\end{aligned}
$$

we have $R(b, \lambda) \sin \left[\alpha_{2}-\theta(b, \lambda)\right]=0$ so $\sin \left[\alpha_{2}-\theta(b, \lambda)\right]=0$, or $\theta(b, \lambda)=\alpha_{2}+(n+1) \pi$. Using by $S(t, \lambda)=$ $\frac{R^{\prime}(t, \lambda)}{R(t, \lambda)}, T(t, \lambda)=\theta^{\prime}(t, \lambda)$ and their asymptotic expansions (15)-(16) we can write

$$
\frac{\sin \alpha_{2}}{\cos \alpha_{2}}=\frac{-\lambda b_{1}^{\prime}+b_{1}+O\left(\eta^{2}(\lambda)\right)+O\left(\lambda \eta^{2}(\lambda)\right)}{-\lambda^{3 / 2} b_{2}^{\prime}+\lambda^{1 / 2} b_{2}+O\left(\eta^{2}(\lambda)\right)+O\left(\lambda \eta^{2}(\lambda)\right)}
$$

so

$$
\begin{aligned}
\tan \alpha_{2} & =\left[\lambda^{-1 / 2} \frac{b_{1}^{\prime}}{b_{2}^{\prime}}-\lambda^{-3 / 2} \frac{b_{1}}{b_{2}^{\prime}}+O\left(\lambda^{-1 / 2} \eta^{2}(\lambda)\right)\right] \times\left[1+\lambda^{-1} \frac{b_{2}}{b_{2}^{\prime}}+O\left(\lambda^{-1 / 2} \eta^{2}(\lambda)\right)\right] \\
& =\lambda^{-1 / 2} \frac{b_{1}^{\prime}}{b_{2}^{\prime}}+O\left(\lambda^{-1 / 2} \eta^{2}(\lambda)\right) .
\end{aligned}
$$

In the last equation, by using Taylor expansion of $\arctan x$ at $x=0$, we obtain

$$
\alpha_{2}=\lambda^{-1 / 2} \frac{b_{1}^{\prime}}{b_{2}^{\prime}}+O\left(\lambda^{-1 / 2} \eta^{2}(\lambda)\right)
$$

Let use these findings (17), (18) and (19) in $\theta(b, \lambda)-\theta(a, \lambda)=\lambda^{1 / 2}(b-a)+\sum_{n=1}^{\infty} \operatorname{Im} \int_{a}^{b} v_{n}(x, \lambda) d x$, we see that

$$
\begin{gathered}
(n+1) \pi+\lambda^{-1 / 2} \frac{b_{1}^{\prime}}{b_{2}^{\prime}}-\frac{a_{1}^{\prime}}{a_{2}^{\prime}} \lambda^{-1 / 2}+\lambda^{-1 / 2} \sin \left(2 \lambda^{1 / 2} a+\xi_{a}\right)-\lambda^{-1} \frac{a_{1}^{\prime}}{a_{2}^{\prime}} \cos \left(2 \lambda^{1 / 2} a+\xi_{a}\right)+O\left(\lambda^{-1 / 2} \eta^{2}(\lambda)\right) \\
=\lambda^{1 / 2}(b-a)+\frac{1}{2} \lambda^{-1 / 2} \sin \left(2 \lambda^{1 / 2} t+\xi_{t}\right)+O\left(\lambda^{-1} \eta(\lambda)\right) .
\end{gathered}
$$

We prove the theorem by using definitions of $\sin \xi_{t}, \cos \xi_{t}$ and $\eta(\lambda)$; also series error computation in the last equation.

\section{Proof of theorem 2.}

(ii) If $a_{2}^{\prime}=0$ and $b_{2}^{\prime}=0$, our problem is

$$
\begin{aligned}
y^{\prime \prime}(t)+\{\lambda-q(t)\} y(t) & =0, \quad t \in[a, b], \\
a_{1} y(a)+a_{2} y^{\prime}(a) & =\lambda a_{1}^{\prime} y(a), \\
b_{1} y(b)+b_{2} y^{\prime}(b) & =\lambda b_{1}^{\prime} y(b) .
\end{aligned}
$$


The real solution of $y^{\prime \prime}(t)+[\lambda-q(t)] y(t)=0$ is $y(t, \lambda)=R(t, \lambda) \cos \theta(t, \lambda)$. We use this equation for boundary $t=a$, we find

$$
R(a, \lambda)\left\{\cos \theta(a, \lambda)\left[a_{2} \frac{R^{\prime}(a, \lambda)}{R(a, \lambda)}-a_{1}^{\prime} \lambda+a_{1}\right]-a_{2} \theta^{\prime}(a, \lambda) \sin \theta(a, \lambda)\right\}=0
$$

If we choose $\alpha_{3}$ as

$$
\begin{aligned}
& \sin \alpha_{3}:=a_{2} \frac{R^{\prime}(a, \lambda)}{R(a, \lambda)}-a_{1}^{\prime} \lambda+a_{1}, \\
& \cos \alpha_{3}:=-a_{2} \theta^{\prime}(a, \lambda),
\end{aligned}
$$

we have $R(a, \lambda) \sin \left(\alpha_{3}+\theta(a, \lambda)\right)=0$ so $\sin \left(\alpha_{3}+\theta(a, \lambda)\right)=0$, or $\theta(a, \lambda)=-\alpha_{3}$. Using by $S(t, \lambda)=\frac{R^{\prime}(t, \lambda)}{R(t, \lambda)}$, $T(t, \lambda)=\theta^{\prime}(t, \lambda)$ and their asymptotic expansions, one writes

$$
\frac{\cos \alpha_{3}}{\sin \alpha_{3}}=\frac{-a_{2} \lambda^{-1 / 2}+a_{2} \cos \left(2 \lambda^{1 / 2} a+\xi_{a}\right)+O\left(\eta^{2}(\lambda)\right)}{-a_{1}^{\prime} \lambda+a_{1}-a_{2} \sin \left(2 \lambda^{1 / 2} a+\xi_{a}\right)+O\left(\eta^{2}(\lambda)\right)}
$$

or

$$
\cot \alpha_{3}=\frac{-a_{2} \lambda^{-1 / 2}+a_{2} \cos \left(2 \lambda^{1 / 2} a+\xi_{a}\right)+O\left(\eta^{2}(\lambda)\right)}{-a_{1}^{\prime} \lambda\left[1-\frac{a_{1}}{a_{1}^{\prime}} \lambda^{-1}+\frac{a_{2}}{a_{1}^{\prime}} \lambda^{-1} \sin \left(2 \lambda^{1 / 2} a+\xi_{a}\right)+O\left(\lambda^{-1} \eta^{2}(\lambda)\right)\right]}
$$

so

$\cot \alpha_{3}=\frac{a_{2}}{a_{1}^{\prime}} \lambda^{-1 / 2}-\frac{a_{2}}{a_{1}^{\prime}} \lambda^{-1} \cos \left(2 \lambda^{1 / 2} a+\xi_{a}\right)+O\left(\lambda^{-1} \eta^{2}(\lambda)\right) \times\left[1+\frac{a_{1}}{a_{1}^{\prime}} \lambda^{-1}-\frac{a_{2}}{a_{1}^{\prime}} \lambda^{-1} \sin \left(2 \lambda^{1 / 2} a+\xi_{a}\right)+O\left(\lambda^{-1} \eta^{2}(\lambda)\right)\right]$

then

$$
\cot \alpha_{3}=\frac{a_{2}}{a_{1}^{\prime}} \lambda^{-1 / 2}-\frac{a_{2}}{a_{1}^{\prime}} \lambda^{-1} \cos \left(2 \lambda^{1 / 2} a+\xi_{a}\right)+\frac{a_{1} a_{2}}{\left[a_{1}^{\prime}\right]^{2}} \lambda^{-3 / 2}-\frac{a_{2}^{2}}{\left(a_{1}^{\prime}\right)^{2}} \lambda^{-3 / 2} \sin \left(2 \lambda^{1 / 2} a+\xi_{a}\right)+O\left(\lambda^{-1} \eta^{2}(\lambda)\right)
$$

In the last equation, by using Taylor expansion of $\operatorname{arccot} x$ at $x=0$, we obtain

$$
\begin{aligned}
-\theta(a, \lambda) & =\alpha_{3}=\frac{\pi}{2}-\frac{a_{2}}{a_{1}^{\prime}} \lambda^{-1 / 2}+\frac{a_{2}}{a_{1}^{\prime}} \lambda^{-1} \cos \left(2 \lambda^{1 / 2} a+\xi_{a}\right)-\frac{a_{1} a_{2}}{\left[a_{1}^{\prime}\right]^{2}} \lambda^{-3 / 2} \\
& +\frac{a_{2}^{2}}{\left(a_{1}^{\prime}\right)^{2}} \lambda^{-3 / 2} \sin \left(2 \lambda^{1 / 2} a+\xi_{a}\right)+\frac{a_{2}^{3}}{3\left(a_{1}^{\prime}\right)^{3}} \lambda^{-3 / 2}+O\left(\lambda^{-1} \eta^{2}(\lambda)\right) .
\end{aligned}
$$

For boundary $t=b$, by using $y(b, \lambda)=R(b, \lambda) \cos \theta(b, \lambda)$, we find

$$
R(b, \lambda)\left\{\cos \theta(b, \lambda)\left[b_{2} \frac{R^{\prime}(b, \lambda)}{R(b, \lambda)}-b_{1}^{\prime} \lambda+b_{1}\right]-b_{2} \theta^{\prime}(b, \lambda) \sin \theta(b, \lambda)\right\}=0 .
$$

If we choose $\alpha_{4}$ as

$$
\begin{aligned}
& \sin \alpha_{4}:=b_{2} \frac{R^{\prime}(b, \lambda)}{R(b, \lambda)}-b_{1}^{\prime} \lambda+b_{1}, \\
& \cos \alpha_{4}:=b_{2} \theta^{\prime}(b, \lambda),
\end{aligned}
$$


we have $R(b, \lambda) \sin \left[\alpha_{4}-\theta(b, \lambda)\right]=0$ so $\sin \left[\alpha_{4}-\theta(b, \lambda)\right]=0$, or $\theta(b, \lambda)=(n+1) \pi+\alpha_{4}$. Using by $S(t, \lambda)=$ $\frac{R^{\prime}(t, \lambda)}{R(t, \lambda)}, T(t, \lambda)=\theta^{\prime}(t, \lambda)$ and their asymptotic expansions, one writes

$$
\begin{aligned}
\cot \alpha_{4} & =\frac{\lambda^{-1 / 2} b_{2}+O\left(\lambda^{-1} \eta^{2}(\lambda)\right)}{-b_{1}^{\prime} \lambda\left[1-\frac{b_{1}}{b_{1}^{\prime}} \lambda^{-1}+O\left(\lambda^{-1} \eta^{2}(\lambda)\right)\right]} \\
& =\left[-\lambda^{-1 / 2} \frac{b_{2}}{b_{1}^{\prime}}+O\left(\lambda^{-2} \eta^{2}(\lambda)\right)\right] \times\left[1+\frac{b_{1}}{b_{1}^{\prime}} \lambda^{-1}+O\left(\lambda^{-1} \eta^{2}(\lambda)\right)\right],
\end{aligned}
$$

then

$$
\cot \alpha_{4}=-\lambda^{-1 / 2} \frac{b_{2}}{b_{1}^{\prime}}-\lambda^{-3 / 2} \frac{b_{1} b_{2}}{\left(b_{1}^{\prime}\right)^{2}}+O\left(\lambda^{-1} \eta^{2}(\lambda)\right) .
$$

In the last equation, by using Taylor expansion of $\operatorname{arccot} x$ at $x=0$, we obtain

$$
\alpha_{4}=\frac{\pi}{2}+\lambda^{-1 / 2} \frac{b_{2}}{b_{1}^{\prime}}+\lambda^{-3 / 2} \frac{b_{1} b_{2}}{\left(b_{1}^{\prime}\right)^{2}}-\lambda^{-3 / 2} \frac{b_{2}^{3}}{3\left(b_{1}^{\prime}\right)^{3}}+O\left(\lambda^{-1} \eta^{2}(\lambda)\right)
$$

so

$$
\theta(b, \lambda)=(n+1) \pi+\frac{\pi}{2}+\lambda^{-1 / 2} \frac{b_{2}}{b_{1}^{\prime}}+\lambda^{-3 / 2} \frac{b_{1} b_{2}}{\left(b_{1}^{\prime}\right)^{2}}-\lambda^{-3 / 2} \frac{b_{2}^{3}}{3\left(b_{1}^{\prime}\right)^{3}}+O\left(\lambda^{-1} \eta^{2}(\lambda)\right)
$$

Let use these findings in $\theta(b, \lambda)-\theta(a, \lambda)=\lambda^{1 / 2}(b-a)+\sum_{n=1}^{\infty} \operatorname{Im} \int_{a}^{b} v_{n}(x, \lambda) d x$, we estimate that

$$
\begin{aligned}
& (n+1) \pi+\frac{\pi}{2}+\lambda^{-1 / 2} \frac{b_{2}}{b_{1}^{\prime}}+\lambda^{-3 / 2} \frac{b_{1} b_{2}}{\left(b_{1}^{\prime}\right)^{2}}-\lambda^{-3 / 2} \frac{b_{2}^{3}}{3\left(b_{1}^{\prime}\right)^{3}} \\
& +\frac{\pi}{2}-\frac{a_{2}}{a_{1}^{\prime}} \lambda^{-1 / 2}+\frac{a_{2}}{a_{1}^{\prime}} \lambda^{-1} \cos \left(2 \lambda^{1 / 2} a+\xi_{a}\right)-\frac{a_{1} a_{2}}{\left[a_{1}^{\prime}\right]^{2}} \lambda^{-3 / 2} \\
& +\frac{a_{2}^{2}}{\left[a_{1}^{\prime}\right]^{2}} \lambda^{-3 / 2} \sin \left(2 \lambda^{1 / 2} a+\xi_{a}\right)+\frac{\left(a_{2}\right)^{3}}{3\left[a_{1}^{\prime}\right]^{3}} \lambda^{-3 / 2}+O\left(\lambda^{-1} \eta^{2}(\lambda)\right) \\
& =\lambda^{1 / 2}(b-a)+\frac{1}{2} \lambda^{-1 / 2} \sin \left(2 \lambda^{1 / 2} t+\xi_{t}\right)+O\left(\lambda^{-1} \eta(\lambda)\right) .
\end{aligned}
$$

We prove the theorem by using definitions of $\sin \xi_{t}, \cos \xi_{t}$ and $\eta(\lambda)$; also series error computation in the last equation.

Similarly, Theorem 1 (ii) follows from (14), (18) and (21); Theorem 2 (i) follows from (14), (20) and (19).

\section{Competing interests}

The authors declare that they have no competing interests.

\section{Authors' contributions}

All authors have contributed to all parts of the article. All authors read and approved the final manuscript. 


\section{References}

[1] Başkaya, E., Regular Sturm-Liouville problems with eigenvalue parameter in the boundary conditions, PhD. Thesis, Karadeniz Technical University, The Graduate School of Natural and Applied Sciences Biology Graduate Program, Trabzon, 2013.

[2] Coskun, H.; Bayram, N., Asymptotics of eigenvalues for regular Sturm-Liouville problems with eigenvalue parameter in the boundary condition, J. Math. Anal. Appl. 306 (2005), 548-566.

[3] Coskun, H.; Harris, B. J., Estimates for the periodic and semi-periodic eigenvalues of Hill's equation, Proc. Roy. Soc. Edinburgh (A) 130 (2000), 991-998.

[4] Coskun, H, On the spectrum of a second-order periodic differential equation, Rocky Mountain J. Math. 33 (2003), 1261-1277.

[5] Eastham, M. S., The Spectral Theory of Periodic Differential Equations, Scottish Academic Press, Edinburgh 1973.

[6] Fix, G., Asymptotic eigenvalues of Sturm-Liouville systems, J. Math. Anal. Appl. 19 (1967), 519-525.

[7] Fulton, C. T., An integral equation iterative scheme for asymptotic expansions of spectral quantities of regular Sturm-Liouville problems, J. Integral Equations 4 (1982), 163-172.

[8] Fulton, C. T., Two point boundary value problems with eigenvalue parameter contained in the boundary conditions, Proc. Roy. Soc. Edinburgh (A) 77 (1977), 293-308.

[9] Fulton, C. T.; Pruess, S. A., Eigenvalue and eigenfunction asymptotics for regular Sturm-Liouville problems, J. Math. Anal. Appl. 182 (1994), 297-340.

[10] B. J. Harris, A series solution for certain Riccati equations with applications to Sturm-Liouville problems, J. Math. Anal. Appl. 137 (1989), 462-470.

[11] B. J. Harris, Asymptotics of eigenvalues for regular Sturm-Liouville problems, J. Math. Anal. Appl. 183 (1994), 25-36.

[12] B. J. Harris, The form of the spectral functions associated with Sturm-Liouville problems with continuous spectrum, Mathematika 44 (1997), 149-161.

[13] Hochstadt, H., Asymptotic estimates for the Sturm-Liouville spectrum, Comm. Pure Appl. Math. 14 (1961), $749-764$.

[14] Titchmarsh, E. C., Eigenfunction Expansions Associated with Second Order Differential Equations I, 2 nd edn., Oxford Univ. Press, Oxford 1962.

[15] Walter, J., Regular eigenvalue problems with eigenvalue parameter in the boundary condition, Math. Z. 153 (1973), $301-312$. 